\title{
Solid-solid and liquid-solid phase equilibria for the restricted primitive model
}

\author{
By BEREND SMIT, KLAAS ESSELINK
}

Shell Research B.V., Koninklijke/Shell-Laboratorium, Amsterdam, PO Box 38000, 1030 BN Amsterdam, The Netherlands

\author{
and DAAN FRENKEL
}

FOM-Institute for A tomic and Molecular Physics, Kruislaan 407, 1098 SJ Amsterdam, The Netherlands

(Received 9 July 1995; revised version accepted 16 August 1995)

\begin{abstract}
A Monte Carlo simulation study is made of the phase diagram of the restricted primitive model and of the solid-liquid and solid-solid phase coexistence curves in particular. At low temperatures, there is liquid-bcc coexistence and with increasing density there is bcc-fcc coexistence. These coexistence curves end in a triple point (liquid-bcc-fcc) above which only liquid-fcc coexistence is observed.
\end{abstract}

\section{Introduction}

The nature of the liquid-vapour critical point of ionic systems is one of the outstanding problems in liquid state physics $\{1-7\}$. Computer simulations have played an important role in testing the quality of the various theoretical descriptions of the liquid-vapour coexistence curve $\{8-13\}$. In contrast, not much attention has been paid to the melting transition. This is not surprising, since the melting behaviour of ionic solids should not differ qualitatively from the melting of other substances.

The aim of the present paper is to map out the melting curve of the most widely studied model for ionic fluids, viz. the restricted primitive model. The critical temperature of this model is quite low and it is not obvious therefore whether the liquid phase is thermodynamically stable. For example, in the case of $\mathrm{C}_{60}$ or colloid polymer mixtures, the triple point is preempted by freezing $\{14,15\}$.

\section{Model}

The restricted primitive model consists of a two-component mixture of hard spheres. All spheres have a diameter $\sigma$ and carry a charge of magnitude $|z e|$, where $e$ is the charge of an electron. To ensure charge neutrality half of the spheres have a positive charge and the other half a negative one. The potential energy between two ions $i$ and $j$ can be written as:

$$
u\left(r_{\mathrm{ij}}\right)=\left\{\begin{array}{lll}
\left(4 \pi \varepsilon \varepsilon_{0}\right) & 1_{z_{\mathrm{i}}} z_{\mathrm{j}} e^{2 / r_{\mathrm{ij}}} & r_{\mathrm{ij}}>\sigma \\
\infty & r_{\mathrm{ij}} \leqslant \sigma
\end{array},\right.
$$

where $r_{\mathrm{ij}}$ is the distance between the two ions $i$ and $j, \varepsilon$ is the dielectric constant of the medium, and $\varepsilon_{0}$ is the permeability of vacuum.

It is convenient to introduce reduced units; the energy is expressed in units 
$(z e)^{2} /\left(4 \pi \varepsilon \varepsilon_{0} \sigma\right)$ and the distance in units $\sigma$. This gives for the unit of temperature $(z e)^{2} /\left(4 \pi \varepsilon \varepsilon_{0} \sigma k_{\mathrm{B}}\right)$, where $k_{\mathrm{B}}$ is Boltzmann's constant. The density is expressed in units of $\sigma^{3}$.

\section{Simulations}

\subsection{Preliminaries}

We assume that the restricted primitive model can be in two stable solid phases: the fcc (face centred cubic) phase and the bcc (body centred cubic) phase.

At high temperatures the Coulombic interactions are less important than the hardsphere repulsion. For the hard-sphere model it is assumed that the stable solid phase has an fcc structure $\{16-18\}$. We therefore expect to observe in the restricted primitive model at sufficiently high temperatures fcc-fluid phase coexistence.

The restricted primitive model can be considered as a model of an ionic salt. $\mathrm{Cs}^{+}$ and $\mathrm{Cl}$ have nearly identical radii, and it can therefore be expected that the crystal structure of the restricted primitive model will be closely related to the $\mathrm{CsCl}$ structure. At low temperatures and zero pressure the crystal structure of $\mathrm{CsCl}$ is the bcc structure $\{19\}$. This can be rationalized by comparing the energies of the fcc and bcc structures. In the fcc structure it is impossible to have each anion 12-fold coordinated with cations while maintaining an electrically neutral unit cell. Therefore, at a given density the energy of the fcc lattice is larger than the energy of the bcc structure, in which each anion is 8-fold coordinated with cations. One can expect the bcc structure to be stable at sufficiently low temperatures and pressures. Note that at high temperatures the entropy becomes dominant. Since at a given density the free volume (the volume spanned by the neighbouring atoms in which an atom is free to move) of the bcc structure is smaller than the corresponding free volume of the fcc structure; the fcc structure has a higher entropy than the bcc structure. We can therefore expect a bcc-fcc transition when the temperature or pressure is increased.

The maximum densities for the bcc and fcc structures are $\rho=3 \sqrt{ } 3 / 4$ and $\rho=\sqrt{ } 2$, respectively. Therefore at sufficiently high pressures the fcc structure will be more stable at any temperature.

The above arguments give a qualitative description of the expected phase diagram of the restricted primitive model. Below, we show how the phase boundaries can be determined quantitatively using computer simulations.

\subsection{Description of the simulations}

To calculate the fluid-solid and solid-solid coexistence curves, we have determined the equations of state and the chemical potential of the various phases at various temperatures $(T=1 \cdot 0,0 \cdot 5,0 \bullet 375,0 \bullet 25,0 \bullet 1,0 \bullet 075,0 \bullet 05$, and $0 \bullet 04)$. The current estimate of the vapour-liquid critical temperatures is $T_{\mathrm{c}}=0 \cdot 057\{20\}$.

All simulations were performed in a cubic simulation box using periodic boundary conditions. The long range interactions were handled using the Ewald summation technique with 'tin-foil' boundary conditions $\{21,22\}$.

Throughout this work we have used the ordinary Monte Carlo technique in various ensembles $\{23\}$. The simulations were performed in cycles. Each cycle consists of some attempts to displace a randomly selected particle, and some attempts to change the volume of the system. At each cycle it is decided with a prescribed probability which type of move is attempted; these probabilities are chosen such that on average per cycle $N_{\text {dis }}$ attempts to displace a particle and $N_{\mathrm{vol}}$ attempts to change 


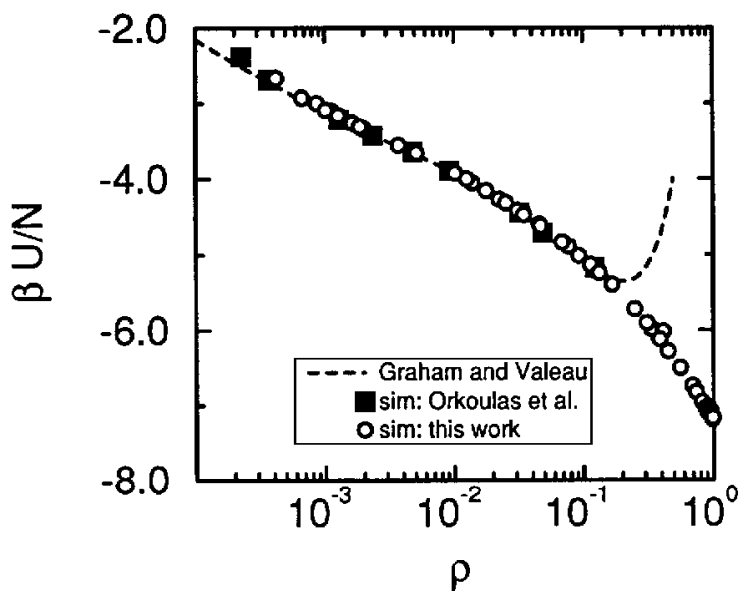

Figure 1. Energy per particle $U / N$ of the restricted primitive model as a function of the density $\rho$; temperature $T=1 / \beta=0 \cdot 1$; a comparison of simulation results of Orkoulas and Panagiotopoulos $\{12\}$, the fit of Graham and Valleau $\{8\}$, and the present work.

the volume are performed. The maximum displacement of a particle was set to a value such that $\sim 30 \%$ of the moves were accepted. For the simulations presented in this work we used $N_{\text {dis }}=250$ and $N_{\text {vol }}=5$. A total simulation consisted of at least 20000 cycles at the highest temperature, and 60000 at the lowest temperature. The first half of every run was used for equilibration and was ignored in the calculation of thermal averages. An estimate of the error was made using the method of Flyvbjerg and Petersen $\{24\}$.

Below we describe the simulation procedure of each phase in detail.

\subsection{Liquid phase}

For the liquid phase the equation of state was determined using standard NPT simulations of a system of 250 particles. The difference between the excess free energy of the liquid at two densities can be obtained by integrating the equation of state:

$$
\frac{\beta \Delta F^{\mathrm{ex}}}{N} \equiv \frac{\beta F^{\mathrm{ex}}(\rho)}{N}-\frac{\beta F^{\mathrm{ex}}\left(\rho_{0}\right)}{N}=\int_{\rho_{0}}^{\rho} \mathrm{d} \rho^{\prime} \frac{\beta P\left(\rho^{\prime}\right)-\rho^{\prime}}{\rho^{\prime 2}},
$$

where $\beta=1 /\left(k_{\mathrm{B}} T\right)$ and the excess free energy is defined as

$$
\frac{\beta F^{\mathrm{ex}}}{N} \equiv \frac{\beta F(\rho)}{N}-\frac{\beta F^{\mathrm{id}}(\rho)}{N}
$$

In this work we define the ideal gas contribution to the free energy to be

$$
\frac{\beta F^{\text {id }}(\rho)}{N} \equiv \ln (\rho / 2)-1,
$$

where $\rho$ is the total number density, and the factor of 2 arises from the fact that we consider a 50:50 mixture.

Equation (2) allows us to determine the free energy at a given density provided that the free energy at density $\rho_{0}$ is known. Normally, $\rho_{0}$ is chosen sufficiently low such that the system behaves like an ideal gas. Here, we use the results of Graham and Valleau $\{8\}$ and Orkoulas and Panagiotopoulos $\{12\}$ to determine $F^{\text {ex }}\left(\rho_{0}\right)$.

For $T=0 \cdot 1$ Orkoulas and Panagiotopoulos $\{12\}$ have determined the excess 


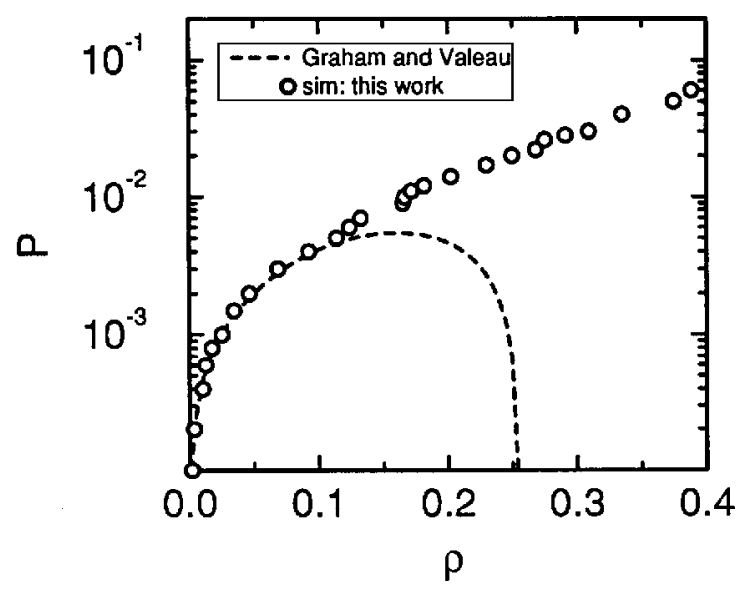

Figure 2. Equation of state of the restricted primitive model $T=0 \cdot 1$; a comparison of simulation results (open symbols) with the fit of Graham and Valleau; $P$ is the pressure and $\rho$ is the density.

chemical potential and energy at low densities $(\rho<0 \bullet 1)$. Orkoulas and Panagiotopoulos have shown that under these conditions their results are in good agreement with the results of Graham and Valleau $\{8\}$. Figures 1 and 2 show that our simulation results for the energy and pressure are in excellent agreement with the results of Orkoulas and Panagiotopoulos and Graham and Valleau. Note that the results of Graham and Valleau are calculated from equation (6) of $\{8\}$; this equation is a fit to the data of Graham and Valleau and is valid only for densities smaller than $\rho=0 \bullet 1$. To calculate the excess free energy we use the low density $(\rho<0 \cdot 09)$ results for the free energy of Graham and Valleau and the equation of state obtained from our simulations for the higher densities.

A check of the consistency of the excess free energy data can be made by comparing the previous calculation with one in which we determine the free energy by calculating the energy as a function of the temperature at a given density from $N V T$ simulations. The free energy difference between a state at temperature $\beta$ and one at temperature $\beta_{0}$ is given by

$$
\frac{\beta F(\beta)}{N}-\frac{\beta_{0} F\left(\beta_{0}\right)}{N}=\int_{\beta}^{\beta_{0}} \mathrm{~d} \beta^{\prime} \frac{U\left(\beta^{\prime}\right)}{N} .
$$

For $\beta=0$ the free energy of the restricted primitive model is equal to the free energy of a hard-sphere fluid, for which the free energy can be calculated from the Carnahan-Starling equation $\{25\}$. In figure 3 the results of this calculation are shown for $\rho=0 \cdot 4$ and $\rho=0 \bullet 6$. These results are in good agreement with the results obtained from the equation of state (2). We use the results of figure 3 to determine $F\left(\rho_{0}\right)$ for the other temperatures. For these temperatures we have to determine the equation of state of the liquid phase for $\rho>0 \cdot 6$.

\subsection{Solid phases}

For the solid phase we have also used standard NPT Monte Carlo simulations to determine the equation of state. The number of particles for the bcc phase was 250 and for the fcc phase 256 .

To determine the absolute free energy of the solid phases, we have used the method of Frenkel and Ladd $\{17\}$. In this method the solid is transformed slowly into an 


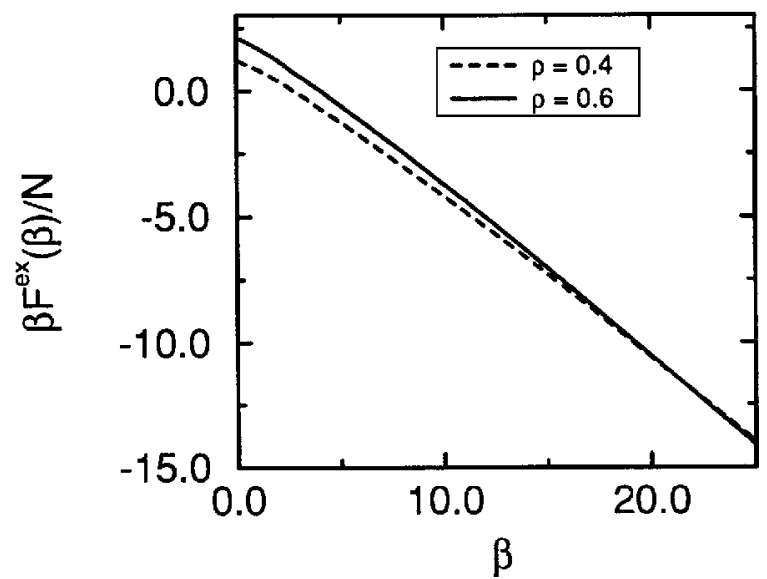

Figure 3. Excess free energy as a function of temperature.

Einstein crystal for which we know the free energy exactly. The potential that can make this transformation is

$$
\tilde{U}\left(\mathbf{r}^{\mathrm{N}}, \lambda\right)=U_{0}+U^{\mathrm{HS}}\left(\mathbf{r}^{\mathrm{N}}\right)+(1-\lambda)\left\{U^{\operatorname{Coul}}\left(\mathbf{r}^{\mathrm{N}}\right)-U_{0}\right\}+\lambda \sum_{\mathrm{i}=1}^{\mathrm{N}} \alpha\left(\mathbf{r}_{\mathrm{i}}-\mathbf{r}_{\mathrm{i}}^{0}\right)^{2},
$$

where $U^{\mathrm{HS}}\left(\mathbf{r}^{\mathrm{N}}\right)$ is the hard-sphere part of the potential (1), $U^{\mathrm{Coul}}\left(\mathbf{r}^{\mathrm{N}}\right)$ the Coulombic part, and $U_{0}$ is the energy of the static lattice. $\mathbf{r}_{\mathrm{i}}^{0}$ is a position of the Einstein lattice assigned to particle $i$ and $\lambda$ is a coupling constant. Note that for $\lambda=0$ we recover the original potential (1). For $\lambda=1$ the Coulombic interactions are 'removed' and we are left with hard spheres that are coupled with harmonic springs (with spring constant $\alpha$ ) to a lattice point $\mathbf{r}_{\mathrm{i}}^{0}$, which is an Einstein crystal. The free energy of the solid of interest can be calculated from the reversible work of transferring this solid into an Einstein crystal. If we use equation (6) as our potential, the free energy difference follows from

$$
\begin{aligned}
\frac{\Delta \beta F_{\mathrm{MC}}}{N} & \equiv \frac{\beta F(\lambda=0)}{N}-\frac{\beta F(\lambda=1)}{N}=\int_{\lambda=1}^{\lambda=0} \mathrm{~d} \lambda\left\langle\frac{\partial \beta \tilde{U}(\lambda) / N}{\partial \lambda}\right\rangle_{\lambda} \\
& =\int_{1}^{0} \mathrm{~d} \lambda\left\langle-\left\{U^{\operatorname{Cou} 1}\left(\mathbf{r}^{\mathrm{N}}\right)-U_{0}\right\}+\sum_{\mathrm{i}=1}^{\mathrm{N}} \alpha\left(\mathbf{r}_{\mathrm{i}}-\mathbf{r}_{\mathrm{i}}^{0}\right)^{2}\right\rangle_{\lambda}
\end{aligned}
$$

To avoid a divergence of the integration for $\lambda \rightarrow 0\{17\}$ it is convenient to perform the simulations in such a way that the position of the centre of mass is fixed. The free energy of an Einstein crystal with fixed centre of mass is given by $\{17\}$

$$
\frac{\beta F_{\mathrm{Fin}}^{\mathrm{CM}}(\alpha)}{N}=\frac{\beta U_{0}}{N}-\frac{3(N-1)}{2 N} \ln (\pi / \alpha \beta)+\frac{3}{2 N} \ln N .
$$

The excess free energy is given by

$$
\frac{\beta F^{\mathrm{ex}}(\beta, \rho)}{N}=\frac{\beta U_{0}}{N}+\frac{\beta \Delta F_{\mathrm{MC}}}{N}+\frac{\beta F_{\mathrm{Fin}}^{\mathrm{CM}}(\alpha)}{N}-\frac{\ln V}{\beta N}-\frac{\beta F^{\mathrm{id}}(\rho)}{N},
$$

where the term $\ln V /(\beta N)$ is a correction term to take in to account that the simulations have been performed with a fixed centre of mass.

Table 1 presents the results of the free energy calculations. The consistency of the calculation has been checked by calculating the free energy via the equation of state; if we use for $F\left(\rho_{0}\right)$ the excess free energy given in table 1, the excess free energy at other 
Table 1. Excess free energy per particle $\left(\beta F^{\mathrm{ex}} / N\right)$ of the fcc and bcc solid phases of the restricted primitive model at density $\rho$ and temperatures $T$. The subscript gives the accuracy of the last $\operatorname{digit}(\mathrm{s})$, e.g., $5 \cdot 33_{2}$ means $5 \cdot 33 \pm 0 \cdot 02$.

\begin{tabular}{|c|c|c|c|c|c|}
\hline \multirow[b]{2}{*}{$T$} & & \multicolumn{4}{|c|}{$\rho$} \\
\hline & & $0 \cdot 95$ & $1 \cdot 0$ & $1 \cdot 1$ & $1 \cdot 2$ \\
\hline $1 \cdot 00$ & fcc & & & $6 \cdot 50_{2}$ & $7 \cdot 78_{1}$ \\
\hline $1 \cdot 00$ & bcc & & $5 \cdot 53_{2}$ & & \\
\hline $0 \cdot 50$ & fcc & & & $5 \cdot 75_{2}$ & $7 \cdot 00_{1}$ \\
\hline $0 \cdot 50$ & bcc & & $4 \cdot 80_{2}$ & $6 \cdot 00_{2}^{2}$ & \\
\hline $0 \cdot 375$ & $\mathrm{fcc}$ & & & $5 \cdot 28_{3}^{2}$ & $6 \cdot 53_{1}$ \\
\hline $0 \cdot 375$ & bcc & $3 \cdot 81_{2}$ & $4 \cdot 29$, & $5 \cdot 44_{4}^{2}$ & \\
\hline $0 \cdot 25$ & fcc & & & $4 \cdot 31_{2}^{4}$ & $5 \cdot 52_{1}$ \\
\hline $0 \cdot 25$ & $\mathrm{bcc}$ & $2 \cdot 81_{2}$ & $3 \cdot 25_{2}$ & & \\
\hline $0 \cdot 1$ & fcc & & & $-0 \cdot 076_{1}$ & $0 \cdot 99_{2}$ \\
\hline $0 \cdot 1$ & bcc & $-1 \cdot 72_{2}$ & $-1 \cdot 40_{1}$ & & \\
\hline $0 \cdot 075$ & fcc & & & $-2 \cdot 53_{2}$ & $-1 \cdot 52_{1}$ \\
\hline $0 \cdot 075$ & bcc & & $-4 \cdot 06_{2}$ & $-3 \cdot 21_{2}^{2}$ & \\
\hline $0 \cdot 05$ & fcc & & $-7 \cdot 42_{2}^{2}$ & $-7 \cdot 41_{1}^{2}$ & $-6 \cdot 56_{1}$ \\
\hline $0 \cdot 05$ & $\mathrm{bcc}$ & & $-9 \cdot 36_{2}^{2}$ & $-8 \cdot 69_{2}$ & \\
\hline $0 \cdot 04$ & fcc & & & $-11 \cdot 11_{2}^{2}$ & $-10 \cdot 34_{2}$ \\
\hline $0 \cdot 04$ & $\mathrm{bcc}$ & & $-13 \cdot 35_{3}$ & $-12 \cdot 80_{2}^{2}$ & \\
\hline
\end{tabular}

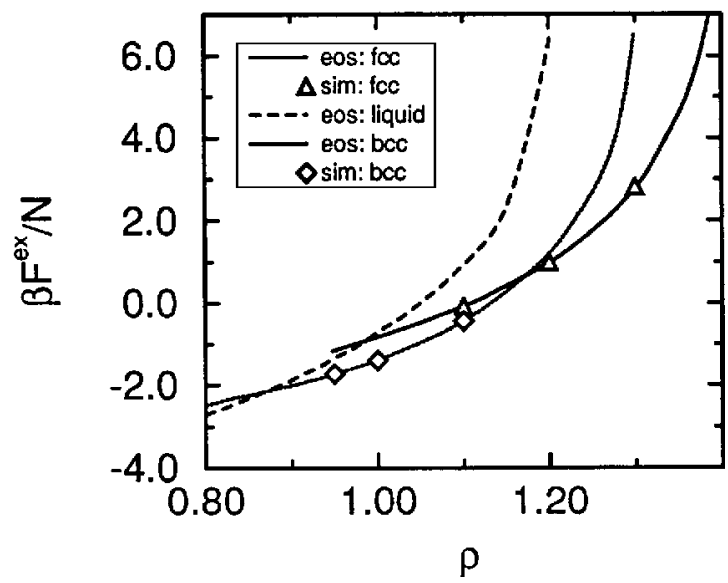

Figure 4. Excess free energy per particle $\beta F^{\mathrm{ex}} / N$ as a function of the density $\rho$ at $T=0 \bullet 1$. The symbols are the results from an absolute free energy calculation, the lines are obtained from the equation of state with one point $\rho_{0}$ for each curve. For comparison the excess free energy of the liquid phase is also shown.

densities can be determined from equation (2). Figure 4 shows the results of this calculation for $T=0 \bullet 1$ for both the fcc and the bcc structures; the free energy data calculated from the equation of state agree very well with the data from table 1 .

\section{Phase diagram}

Having thus obtained the pressure and chemical potential of the liquid and solid phases, the coexistence curve follows by equating the chemical potentials and the pressures of the two phases. The calculated phase diagram is shown in figure 5. At high 


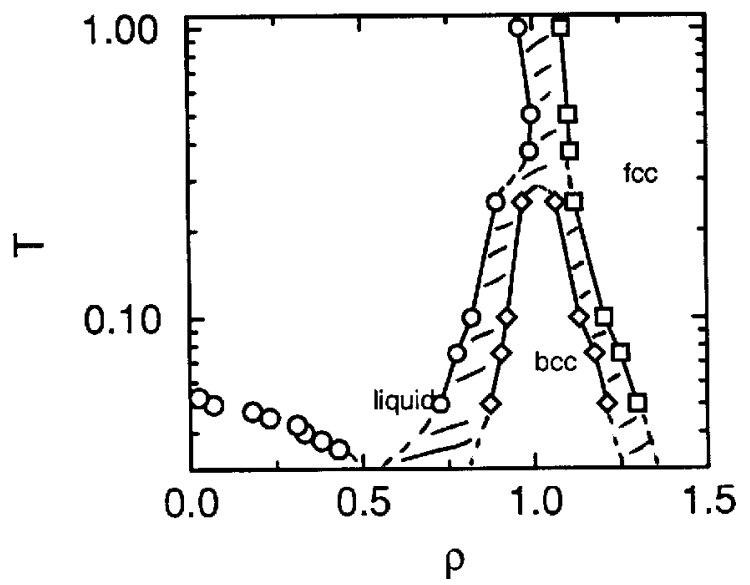

Figure 5. Phase diagram of the restricted primitive model; the data for the liquid coexistence densities are taken from $\{12\}$.

temperatures we observe fluid-fcc coexistence. The fluid-fcc line ends at a triple point where we find three-phase coexistence (fluid-bcc-fcc). This occurs at a temperature $T \approx 0 \cdot 3$. The temperature range of our simulations is not sufficiently low to estimate the vapour-liquid-bcc triple point.

The triple point is located outside the temperature range that we could conveniently study. A rough estimate is $T_{\mathrm{TR}} \approx 0 \cdot 025$. The important point to note is that the triple point is located well below the best estimate of the liquid-vapour critical point. Hence our simulations indicate that freezing does not preempt the liquid-vapour transition of this model system.

This information makes it possible to give a simple prediction of the phase diagram of simple ionic crystals with a $\mathrm{CsCl}$ ground state structure. It would also provide a convenient check for density functional theories of dense ionic systems.

The work of the FOM Institute is part of the research program of FOM and is supported by 'Nederlandse Organisatie voor Wetenschappelijk Onderzoek' (NWO).

\section{References}

\{1\} Fisher, M. E., and Levin, Y., 1993, Phys. Rev. Lett., 71, 3826.

\{2\} González-Tovar, E., and Outhwaite, C. W., 1994, Molec. Phys., 83, 1273.

33\} Leote De Caravalho, R. J. F., and Evans, R., 1994, Molec. Phys., 83, 619.

\{4\} Weingärtner, H., Kleemeier, M., Wiegand, S., and Schröer, W., 1995, J. statist. Phys., 78, 169.

\{5\} Stell, G., 1995, J. statist. Phys., 78, 197.

\{6\} Singh, R. R., and Pitzer, K. S., 1990, J. chem. Phys., 92, 6775.

\{7\} Levelt Sengers, J. M. H., and Given, J. A., 1993, Molec. Phys., 80, 899.

\{8\} Graham, I. S., and Valleau, J. P., 1990, J. phys. Chem., 94, 7894.

\{9\} Valleau, J. P., 1991, J. chem. Phys., 95, 584.

$\{10\}$ Orkoulas, G., and Panagiotopoulos, A. Z., 1993, Fluid Phase Equilibria, 93, 223.

\{11\} Caillol, J.-M., 1994, J. chem. Phys., 100, 2161.

$\{12\}$ Orkoulas, G., and Panagiotopoulos, A. Z., 1994, J. chem. Phys., 101, 1452.

\{13\} Guissani, Y., and Guillot, B., 1994, J. chem. Phys., 101, 490.

\{14\} Hagen, M. H. J., Meijer, E. J., Mooij, G. C. A. M., Frenkel, D., and Lek kerkerker, H. N. W., 1993, Nature (Lond.), 365, 425.

\{15\} Meijer, E. J., and Frenkel, D., 1994, J. chem. Phys., 100, 6873.

\{16\} Hoover, W. G., and Ree, F. H., 1968, J. chem. Phys., 49, 3609. 
\{17\} Frenkel, D., and Ladd, A. J. C., 1984, J. chem. Phys., 81, 3188.

\{18\} Curtin, W. A., and Runge, K., 1987, Phys. Rev. A, 35, 4755.

\{19\} Pauling, L., 1960, The Nature of the Chemical Bond (Ithaca, NY: Cornell University Press).

\{20\} Fisher, M. E., 1994, J. statist. Phys., 75, 1.

\{21\} De Leeuw, S. W., Perram, J. W., and Smith, E. R., 1980, Proc. R. Soc. Lond. A, 373, 27.

\{2\} Esselink, K., 1995, Large Scale Simulations of Many-Particle Systems, Ph.D. thesis, Rijksuniversiteit Groningen, The Netherlands; Esselink, K., 1995, Comput. Phys. Commun., 87, 375.

\{23\} Allen, M. P., and Tildesley, D. J., 1987, Computer Simulation of Liquids (Oxford: Clarendon).

\{24\} Flyvbjerg, H., and Petersen, H. G., 1989, J. chem. Phys., 91, 461.

\{25\} Carnahan, N. F., and Starling, K. E., 1969, J. chem. Phys., 51, 635. 